\title{
A NOTE ON GENERATORS OF SEMIGROUPS
}

\author{
SZE-KAI TSUI
}

\begin{abstract}
The generator $T$ of a norm-continuous semigroup of identity preserving positive linear mappings on a $C^{*}$-algebra $\mathfrak{A}$ is characterized as one that satisfies $T\left(u^{*} u\right) \geqslant u^{*} T(u)+T\left(u^{*}\right) u$ for all unitary elements $u$ in צ.
\end{abstract}

1. Introduction. Since 1948 the analytical theory of semigroups has made vigorous progress. In that year K. Yosida and E. Hille (see [2, p. 363]) independently characterized the infinitesimal generators for strongly continuous semigroups of contractions on a normed linear space as those densely defined closed linear operators $T$ with $\left\|(\lambda I-T)^{-1}\right\|<\lambda^{-1}$ for all $\lambda>0$. Later in 1960 G. Lumer and R. S. Phillips [5] found another description of those generators as those densely defined "dissipative" operators $T$ with range $[I-T]=X$, where $X$ is a semi-inner-product space. If the semigroup of contractions $\alpha(t)$ is norm-continuous, its infinitesimal generator becomes just bounded dissipative. In this note we are concerned with the norm-continuous semigroups of identity preserving positive linear mappings on a $C^{*}$-algebra and find a new characterization of their infinitesimal generators in terms of the $C^{*}$-algebra ordering. It would be more desirable to have a description of the generators of strongly continuous semigroups of positive (or completely positive) linear mappings, and that is currently being investigated by this author.

Lindblad's recent work [3] on generators of one-parameter semigroups of completely positive identity preserving linear mappings on a $C^{*}$-algebra showed an analogous result. As it is known that "positive" case does not follow automatically from "completely positive" case, one part of the proof of Theorem 1 is the same as that used in [3] and the other part is quite different.

2. Preliminary. Let $X$ be a Banach space, $X^{*}$ its dual space and $B(X)$ the Banach algebra of all bounded linear operators (or mappings) on $X$. Given a linear functional $f$ on $X$ and an element $x$ in $X$, we define a linear functional $(x, f)$ on $B(X)$ by $(x, f)(T)=f(T(x))$ for $T$ in $B(X)$. It is easily seen that (i) $\|(x, f)\| \leqslant\|x\|\|f\|$, (ii) $(\alpha x, f)=\alpha(x, f)$ for scalar $\alpha$, (iii) $\left(x_{1}+x_{2}, f\right)=$

Presented to the Society, January 29, 1977; received by the editors August 9, 1976 and, in revised form, January 11, 1977.

AMS (MOS) subject classifications (1970). Primary 46L05; Secondary 22A25.

Key words and phrases. Semigroups of contractions, infinitesimal generators, spatial numerical range, numerical range, dissipative operators, $C^{*}$-algebras, positive linear mappings. 
$\left(x_{1}, f\right)+\left(x_{2}, f\right)$, (iv) $\left(x, f_{1}+f_{2}\right)=\left(x, f_{1}\right)+\left(x, f_{2}\right)$.

The spatial numerical range $V(T)$ of a given linear mapping $T$ in $B(X)$ is defined as

$$
V(T)=\{(x, f)(T) \mid f(x)=1=\|f\|=\|x\|\} .
$$

Let $\mathscr{B}$ be a unital Banach algebra. Given $f$ in $\mathscr{B}^{*}$ and $x$ in $\mathscr{B}$, we define a linear functional on $\mathscr{B}$ as $f_{x}(y)=f(y x)$ for all $y$ in $\mathscr{B}$ and observe (i) $\left\|f_{x}\right\| \leqslant\|f\|\|x\|$, (ii) $f_{\alpha x}=\alpha f_{x}$ for scalar $\alpha$, (iii) $f_{x_{1}+x_{2}}=f_{x_{1}}+f_{x_{2}}$.

The numerical range $V(T, \mathscr{B})$ of a given element $T$ in $\mathscr{B}$ is defined as

$$
V(T, \mathscr{B})=\{f(T) \mid f(I)=1=\|f\|\},
$$

where $I$ is the identity element in $\mathscr{B}$. It is known that $V(T, \mathscr{B})=$ $\left\{f_{x}(T) \mid f(x)=1=\|f\|=\|x\|\right\}$. In the following we list several properties needed in this note whose proofs can be found in [1] (see Theorem 4, p. 84, Theorem 4, p. 30, Theorem 4, p. 28 and Theorem 6, p. 30) and [4].

Proposition 1. $\overline{\text { co }} V(T)=V(T, \mathscr{B})$, where $\overline{\text { co }} V(T)$ is the closed convex hull of $V(T)$. As a consequence $V(T, B)$ is always a closed convex subset in the complex plane $\mathbf{C}$.

Proposition 2.

$$
\begin{aligned}
\operatorname{Max}\{\operatorname{Re} \alpha ; \alpha \in V(T, \mathscr{B})\} & =\inf _{\alpha>0} \frac{1}{\alpha}\{\|I+\alpha T\|-1\} \\
& =\lim _{\alpha \rightarrow 0^{+}} \frac{1}{\alpha}\{\|I+\alpha T\|-1\} .
\end{aligned}
$$

Proposition 3.

$$
\begin{aligned}
\operatorname{Max}\{\operatorname{Re} \alpha ; \alpha \in V(T, \mathscr{B})\} & =\sup _{\alpha>0} \frac{1}{\alpha}\{\log \|\exp (\alpha T)\|\} \\
& =\lim _{\alpha \rightarrow 0^{+}} \frac{1}{\alpha}\{\log \|\exp (\alpha T)\|\} .
\end{aligned}
$$

Proposition 4. Let $x$ be in $\mathscr{B}$. Then $\operatorname{Re} \lambda \leqslant 0$ for all $\lambda$ in $V(x, \Re)$ if and only if $\|\exp (t x)\| \leqslant 1$ for all $t \geqslant 0$.

Definition. An element $x$ in $\mathscr{B}$ is said to be dissipative if $\operatorname{Re} \lambda \leqslant 0$ for all $\lambda$ in $V(x, \mathscr{B})$.

A linear mapping $T$ of a $C^{*}$-algebra $\mathfrak{A}$ into itself is called selfadjoint if $T\left(x^{*}\right)=T(x)^{*}$ for all $x$ in $\mathfrak{A}$, and is called positive if $T(x)$ is positive for all positive elements $x$ in $\mathfrak{A}$. It is easy to see that a positive linear mapping is also selfadjoint.

3. The main theorem. Let $e^{t T}$ be a one-parameter norm-continuous semigroup of positive linear mappings on a $C^{*}$-algebra $\mathscr{A}$ with $e^{t T}(1)=1$, and $T$ its generator $\left(1\right.$ is the identity element in $\mathfrak{A}$, and all $C^{*}$-algebras considered in this note have identity element). From $T(x)=\lim _{t \rightarrow 0^{+}}\left(e^{t T}(x)\right.$ $-x) / t(x \in \mathfrak{Q})$, we see that $T$ is selfadjoint and $T(1)=0$. In the following is 
the theorem in which the generator $T$ is characterized by $C^{*}$-algebra ordering structure.

THEOREM 1. Let $T$ be a bounded selfadjoint linear mapping on a $C^{*}$-algebra $\mathfrak{A}$ with $T(1)=0$. The following two conditions are equivalent:

(i) $T$ is dissipative.

(ii) $T\left(u^{*}\right) u+u^{*} T(u) \leqslant 0$ for all unitary elements $u$ in $\mathfrak{A}$.

Proof. (ii) $\Rightarrow$ (i). The argument used here is the same as that in Proposition 4 in [3]. For the sake of completeness we do it as below. Because of Proposition 2 and a theorem due to Russo and Dye [6], it suffices to show that

$$
r(T) \equiv \lim _{t \rightarrow 0^{+}} \sup _{u} \frac{1}{t}\{\|u+t T(u)\|-1\} \leqslant 0,
$$

where the supremum is taken over all unitary elements in $\mathscr{A}$.

$$
\begin{aligned}
\|u+t T(u)\|^{2} & =\left\|u^{*} u+u^{*} t T(u)+t T\left(u^{*}\right) u+t^{2} T\left(u^{*}\right) T(u)\right\| \\
& \leqslant\left\|1+t^{2} T\left(u^{*}\right) T(u)\right\| \leqslant 1+t^{2}\|T\|^{2} .
\end{aligned}
$$

Hence, $\|I+t T\|^{2} \leqslant 1+t^{2}\|T\|^{2}$, and

$$
(\|I+t T\|-1)(\|I+t T\|+1)<t^{2}\|T\|^{2},
$$

hence,

$$
(\|I+t T\|-1) / t \leqslant t\|T\|^{2} /(\|I+t T\|+1) .
$$

Therefore $r(T) \leqslant 0$.

(i) $\Rightarrow$ (ii). By a theorem due to Russo and Dye [6] we have

$$
\begin{aligned}
\|I+t T\|^{2} & =\sup _{\substack{u: \text { unitary } \\
\text { in } \mathscr{I}}}\|(I+t T)(u)\|^{2} \\
& =\sup _{u}\left\|(u+t T(u))^{*}(u+t T(u))\right\| .
\end{aligned}
$$

Hence, for any state $\phi$ on $\mathfrak{A}$ and unitary element $u$ in $\mathfrak{A}$, we have

$$
\begin{gathered}
\phi\left((u+t T(u))^{*}(u+t T(u))\right) \leqslant\|I+t T\|^{2}, \\
\phi\left(u^{*} u+t\left(T\left(u^{*}\right) u+u^{*} T(u)\right)+t^{2} T\left(u^{*}\right) T(u)\right) \leqslant\|I+t T\|^{2}, \\
t \phi\left(T\left(u^{*}\right) u+u^{*} T(u)\right)+t^{2} \phi\left(T\left(u^{*}\right) T(u)\right) \leqslant\|I+t T\|^{2}-1, \\
\phi\left(T\left(u^{*}\right) u+u^{*} T(u)\right)+t \phi\left(T\left(u^{*}\right) T(u)\right) \\
\leqslant((\|I+t T\|-1) / t)(\|I+t T\|+1) .
\end{gathered}
$$

Taking the limit for both sides as $t \rightarrow 0^{+}$we have

$$
\phi\left(T\left(u^{*}\right) u+u^{*} T(u)\right) \leqslant r(T) \cdot 2 \leqslant 0
$$

because $r(T) \leqslant 0$. Therefore $T\left(u^{*}\right) u+u^{*} T(u) \leqslant 0$. Q.E.D.

COROLlaRY 1. The generator $T$ of a norm-continuous semigroup of identity preserving positive linear mappings on a $C^{*}$-algebra satisfies condition (ii) in Theorem 1. 
Proof. It is because of Proposition 4 and Theorem 1. Q.E.D.

Without the assumption $T(1)=0$ in the above corollary, condition (ii) can be replaced by

(ii) $T(1)+u^{*} T(1) u-T\left(u^{*}\right) u-u^{*} T(u) \geqslant 0$, for all unitary $u$ in $\mathfrak{A}$. Define $T^{\prime}(x)=T(x)-\frac{1}{2}(T(1) x+x T(1))$ for all $x$ in $\mathfrak{X}$. Since

$$
T^{\prime}\left(u^{*}\right) u+u^{*} T^{\prime}(u)=T\left(u^{*}\right) u+u^{*} T(u)-u^{*} T(1) u-T(1),
$$

condition (ii) holds for $T^{\prime}$ if and only if condition (ii)' holds for $T$. Denote $x \rightarrow K x+x K$ by $\{K, x\} \equiv T_{K}(x)$. The semigroup generated by $T_{K}$ is $\exp t T_{K}(x)=e^{t K} x e^{t K}$. The semigroup generated by $T^{\prime}+T^{\prime \prime}(=T)$, where $T^{\prime \prime}=T_{(1 / 2) T(1)}$, is given by the Lie-Trotter formula

$$
\exp t\left(T^{\prime}+T^{\prime \prime}\right)=\lim _{n \rightarrow \infty}\left[\exp \left(t T^{\prime} / n\right) \exp \left(t T^{\prime \prime} / n\right)\right]^{n} \text {. }
$$

Hence $\exp t\left(T^{\prime}+T^{\prime \prime}\right)$ is positive if $T$ satisfies (ii) ${ }^{\prime}$. Conversely, suppose that $\exp t T$ is positive. Then $T$ is selfadjoint. By the Lie-Trotter formula the semigroup generated by $T-T^{\prime \prime}\left(=T^{\prime}\right)$ is positive, and $\left(\exp t T^{\prime}\right)(1)=1$ for all $t>0$. By Corollary 1, condition (ii) holds for $T^{\prime}$. Therefore condition (ii) holds for $T$. We have concluded

CoRollaRY 2. Let $T$ be a bounded selfadjoint linear mapping from $\mathfrak{A}$ into itself. Then $\exp t T$ is positive for all $t \geqslant 0$ iff condition (ii)' holds for $T$.

\section{REFERENCES}

1. F. F. Bonsall and J. Duncan, Numerical ranges of operators on normed spaces and of elements of normed algebras, Cambridge Univ. Press, New York, 1971.

2. E. Hille and R. S. Phillips, Functional analysis and semi-groups, Amer. Math. Soc. Colloq. Publ., vol. 31, Amer. Math. Soc., Providence, R.I., 1957.

3. G. Lindblad, On the generators of quantum dynamical semigroups, Comm. Math. Phys. (to appear).

4. G. Lumer, Semi-inner-product spaces, Trans. Amer. Math. Soc. 100 (1961), $29-43$.

5. G. Lumer and R. S. Phillips, Dissipative operators in a Banach space, Pacific J. Math. 11 (1961), 679-698.

6. B. Russo and H. A. Dye, A note on unitary operators in $C^{*}$-algebras, Duke Math. J. 33 (1966), 413-416.

Department of Mathematical Sciences, OAkland University, Rochester, Michigan 48063 